\title{
Access Rule Consistency in Cooperative Data Access Environment
}

\author{
Meixing Le, Krishna Kant, Sushil Jajodia \\ Center for Secure Information Systems \\ George Mason University, Fairfax, VA \\ $\{$ mlep, kkant, jajodia $\} @$ gmu.edu
}

\begin{abstract}
In this paper we consider the situation where a set of enterprises need to collaborate to provide rich services to their clients. Such collaboration often requires controlled access to each other's data, which we assume is stored in standard relational form. The access control is provided by a set of access rules that may be defined over the joins of various relations. In this paper we introduce the notion of consistency of access rules and devise an algorithm to ensure consistency. We also consider the possibility of occasional changes in access rules and address the problem of maintaining consistency in the face of such changes. We propose algorithms for both changes with new privileges grants and revocations on existing privileges.
\end{abstract}

Index Terms-access rule consistency, cooperative data access, join path

\section{INTRODUCTION}

Providing rich services to clients with minimal manual intervention or paper documents requires the enterprises involved in the service path to collaborate and share data in an orderly manner. For instance, an automated determination of patient coverage and costs requires that a hospital and insurance company be able to make certain queries against each others' databases. Similarly, to arrange for automated shipping of merchandise and to enable automated status checking, the ecommerce vendor and shipping company should be able to exchange relevant information, perhaps in form of database queries. In such environments, data must be released only in a controlled way among cooperative parties, subject to the authorization policies established by them. In this paper, we expose and study various issues that arise in such a collaboration.

In general, enterprise data may appear in a variety of forms, including the simplistic key-value forms like Google's BigTable. However, for concreteness, we assume that all data is stored in relational form, with all tables in a standard normal form. In such a model, data access privileges are given by a set of access rules, each of which is defined either on original tables belonging to an enterprise or over the lossless joins of two or more of these. The join operations, coupled with appropriate projection and selection operations define the access restrictions; although in order to enable working with only the schemas, we ignore selection operation.

This material is based upon work supported by the National Science Foundation under grant CT-20013A and by the US Air Force Office of
A query is authorized only if there is a given access rule providing enough privileges. However, as the access rules are defined on the join results of basic relations, a party can get information from several cooperative parties and perform local computation to obtain the result that is not authorized by any rule. To give a simple example, if an enterprise $P$ is authorized to get relations $R$ and $S$ from parties $P_{R}$ and $P_{S}$ respectively, then it can obtain the result of $R \bowtie S$ (over appropriate join attribute). As the access rules are defined according to business requirements among the cooperative parties, it is possible that there is no rule authorizing $P$ to access the join result of $R \bowtie$ $S$. Consequently, there is a conflict among the access rules. To avoid such conflict, one solution is to add an additional privileges to allow $P$ to access the join result. In contrast, the alternative way is to constrain the party $P$ so that it cannot access both $R$ and $S$ at the same time and local computation is prevented.

In this work, we explore the first approach, and remove the conflicts among the rules by adding more access rules. Given a set of access rules, we propose an algorithm to generate additional rules so as to remove all the conflicts. When a new rule is added, we need to further consider the new conflicts caused by this rule. To achieve that, the algorithm takes advantage of the functional dependencies among the basic relations to add all needed rules. Although the worst case complexity of the algorithm is exponential, the real world complexity is generally quite acceptable due to fact that chains of joins are rare in practice. In addition, the evaluation can be done as pre-computation so that its complexity is not critical.

Since the business relationships among the cooperative parties may change from time to time, the access rules also change correspondingly. We consider two types of changes on the access rules: independent change and cooperative change. The first type of change only affects the rules on a single party and the latter one involves multiple parties. Since any changes on the access rules may result in new conflicts, we also propose algorithms to remove conflicts in the cases of a new access rule is granted or an existing rule is revoked. In both cases, a single change can lead to a series of changes in order to ensure consistency. For the cooperative access rule changes, we assume that the enterprises negotiate and agree to the necessary changes in advance. This means that the actual changes must be introduced simultaneously for all the parties. We propose a mechanism to deal with the required synchronization in this case. The main issue to address is to 
ensure that rule changes are introduced in such a way that minimum number of queries are affected.

The outline of the paper is as follows. Section II addresses the issue of consistency of rules in a cooperative access environment and Section III describes an algorithm for consistency checking. Section IV deals with the problem of changes in the rules. Section V discusses the related work. Finally, Section VI concludes the discussion and lays out areas for future work.

\section{Consistency of AcCess Rules}

\section{A. preliminaries}

We consider a group of cooperating parties, each of which maintains its data in a standard relational form such as Boyce-Codd Normal Form (BCNF). It is possible to consider more complex normal forms as well, but this is beyond the scope of this paper. We also assume simple select-project-join queries, i.e., no cyclic join schemas or queries. The query may be answered by any of the parties that has the required permissions. We assume that the join schema is given - i.e., all the possible join attribute sets between any two relations are known. Each join in the schema is lossless so that a join attribute is always a key attribute of some relations. We also assume that the rules are composeable, which means each rule has all the key attributes of the basic relations in its join path. We study the problems only involving the cooperating enterprises; no "helper" third parties are considered here.

Each cooperative party is given a set of access rules that are defined over the join results of basic relations owned by these parties. We call a sequence of joins as a join path. An access rule is further defined with the attribute set authorized on a specified join path.

Definition 1: A join path is the result of a series of join operations over a set of relations $R_{1}, R_{2} \ldots R_{n}$ with the specified equi-join predicates $\left(A_{l 1}, A_{r 1}\right),\left(A_{l 2}, A_{r 2}\right) \ldots\left(A_{l n}, A_{r n}\right)$ among them, where $\left(A_{l i}, A_{r i}\right)$ are the join attributes from two relations. We use the notation $J_{t}$ to indicate the join path of rule $r_{t}$. We use $J R_{t}$ to indicate the set of relations in a join path $J_{t}$. The length of a join path is the cardinality of $J R_{t}$.

An access rule $r_{t}$ is a triple $\left[A_{t}, J_{t}, P_{t}\right]$, where $J_{t}$ is called the join path of the rule, $A_{t}$ is the set of authorized attributes, and $P_{t}$ is the party authorized to access these attributes. (Note that projection over the authorized set of attributes is implicit here but the order of joins in an actual implementation may be done according to performance considerations.) Each access rule defines a new relation, and we can perform the relational operations such as join on them as well. Correspondingly, a query $q$ can be represented as a pair $\left[A_{q}, J_{q}\right]$, and any party has the authorized rule can answer the query.

\section{B. A running example}

Our running example models an e-commerce scenario with five parties: (a) E-commerce, denoted as $E$, is a company that sells products online, (b) Customer_Service, denoted $C$, is another entity that provides customer service functions (potentially for more than one company), (c) Shipping, denoted $S$, provides shipping services (again, potentially to multiple companies), (d) Supplier, denoted $P$, is the party that stores products in the warehouses, and finally (e) Warehouse, denoted $W$, is the party that provides storage services. To keep the example simple, we assume that each party has but one relation for its local database described below. The attributes should be self-explanatory; the key attributes are indicated by underlining them. In each of these relations, a single attribute happens to form the key, but this is not required in our analysis.

1) E-commerce (order_id, product_id, total) as E

2) Customer_Service (order_id, issue, assistant) as C

3) Shipping (order_id, address, delivery_type) as $\mathrm{S}$

4) Warehouse (product_id, supplier_id, location) as W

5) Supplier (supplier_id, supplier_name, factory) as $P$

In the following, we use oid to denote order_id for short, pid stands for product_id, sid stands for supplier_id, and delivery stands for delivery_type. The possible join schema is also given in figure 1. Relations $E, C, S$ can join over their common attribute oid; relation $E$ can join with $W$ over the attribute pid, and $W$ can join with $P$ on sid. In the example, relations are in $\mathrm{BCNF}$, and the only functional dependency (FD) in each relation is the one implied by the key attribute (i.e., key attribute determines everything else).

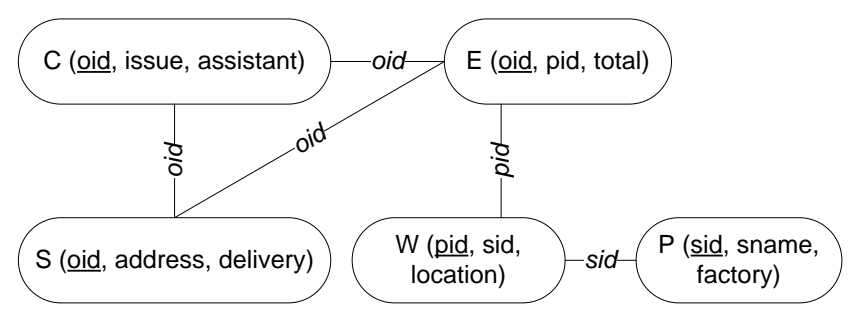

Fig. 1. The given join schema for the example

We now define a set of access rules given to the party $E$ as described in Table I. (Suitable rules must also be defined for other parties, but are not shown here for brevity.) The first column of the table is the rule numbers, and the second column shows the attribute sets of the rules. The third column lists the join paths on which the rules are defined. The last column (redundant in this example) indicates the party to which the rules are given.

\begin{tabular}{|c|l|l|l|}
\hline Rule No. & Authorized attribute set & Join Path & Party \\
\hline 1 & $\{$ oid, pid, total & $E$ & $P_{E}$ \\
\hline 2 & $\{$ oid, issue, address & $S \bowtie_{\text {oid }} C$ & $P_{E}$ \\
\hline 3 & \{oid, pid, total, issue & $E \bowtie_{\text {oid }} C$ & $P_{E}$ \\
\hline 4 & \{oid, pid, sid, location, total $\}$ & $E \bowtie_{\text {pid }} W$ & $P_{E}$ \\
\hline 5 & \{pid, sid, factory & $W \bowtie_{\text {sid }} P$ & $P_{E}$ \\
\hline
\end{tabular}

TABLE I

ACCESS RULES FOR E-COMMERCE COOPERATIVE DATA ACCESS

\section{Rule conflicts and consistency}

There are two styles in which rules can be given. An implicit specification means any valid compositions of the given rules are also considered as valid rules. In contrast, an explicit specification lists out all the allowed accesses and any access not included in the list is not allowed. Given our chosen method of conflict resolution (i.e., by adding rules), 
the distinction between implicit and explicitly specification is not significant, as we shall see shortly.

For a query $q$ to be authorized by explicit rules, there must be an access rule $r_{t}$ whose join path $J_{t}$ is equivalent to $J_{q}$ and $A_{t}$ is a superset of $A_{q}$. In general, it is possible that a party obtains two pieces of information, say $R$ and $S$ according to two different explicit rules. It is then free to join these locally and obtain $R \bowtie S$ even if no rule authorizes access to this composition. Such a situation creates a conflict since access to $R \bowtie S$ is not allowed by the rules but is still possible. We say the set of rules are inconsistent if an access conflict exists with respect to any join path. As stated earlier, the inconsistency can be removed in one of two ways: (a) By adding additional rules that allow for all potential compositions that have not been explicitly specified, or (b) by actually denying access to $R \bowtie S$. The latter can be done via revocation of some existing rules. For instance, we can revoke the privilege of the party $P$ to access $R$, so that the local computation of $R \bowtie S$ is not possible. However, as these initial rules are given according to their business requirements, it is not desired to completely revoke such rules as it may affect the business interactions. Alternatively, we can use the well-known Chinese Wall policy [4] whereby the party can either access $R$ or $S$ but not both simultaneously (and hence cannot compute $R \bowtie S$ ). In this paper we adopt solution (a). In most cases, it is reasonable to allow the local computation results once the underlying information is authorized. For this, one must generate all possible compositions of the given rules and add any missing ones from the list. Therefore, whether we start out with an implicit or explicit specification, the result will be the same. We now define the notion of closure to make the rules consistent.

Definition 2: If two rules $r_{i}, r_{j}$ of party $P$ can be joined losslessly according to the given join schema, and the resulting information $\left[A_{i} \cup A_{j}, J_{i} \bowtie J_{j}\right]$ is also authorized by another rule $r_{k}$ of party $P$, then we say the two rules are "upwards closed". For a set of rules, if any two rules that can be joined losslessly are "upwards closed", we say the set of rules is consistent, and the rules form a consistent closure.

As access rules are usually defined by the parties based on their business needs, the given set of rules is usually inconsistent. Therefore, it is desired to have a mechanism to add the necessary rules so as to make the rule set a consistent closure. Although we are discussing the problem in a cooperative environment, the rule consistency property applies to each individual party separately. Thus, the mechanism for achieving consistent closure below only involves rules on one party.

\section{Key attributes hierachy}

Since we assume all the basic relations are in BCNF, and the join paths are the results of lossless join operations, the key attributes of basic relations in the given join schema form a hierarchal relationship. For instance, suppose that the relations $R, S$ have their key attributes $R . K$ and $S . K$ respectively. If these relations can join losslessly, then the joining attribute must be the key attribute in at least one of them [2]. That is, either the join is performed on R.K, S.K, or R.K is the same attribute as $S . K$. In either case, one key attribute from a basic relation is also the key attribute of the join result of the two relations. Therefore, if the join is performed over the attribute $S . K(R . K \neq S . K)$, then the attribute $R . K$ can functionally determine the relation $S$. In such case, we say $R . K$ is at a higher level than $S . K$, denoted $R . K \rightarrow S . K$. If $R . K=S . K$, there is no hierarchy, and such key attribute of $R$ and $S$ is also the key attribute of the join result. Thus, for a given valid join path, the key attribute of the join path is a key attribute from a basic relation. We call the key attribute of the join path in an access rule as key of the rule. Also, the join attributes in the join paths are always key attributes of some basic relations so these join attributes form the hierarchal relationship. For instance, in the running example, the key attribute oid is at the top level, and we have the hierarchal relationship for three key attributes, where oid $\rightarrow$ pid $\rightarrow$ sid.

For each key attribute of basic relation, we create a group for the rules that take this attribute as their key attribute. As the rules within this group share the same key attribute, any two of them can join over their key attributes.

Definition 3: A join group is a group of access rules associated with a key (join) attribute, where all the attributes in these rules functionally depend on this attribute. If a join group is consistent, then it is called a consistent join group.

Since some rules can be the result of local computation over other rules, there also exist relationships among the rules. In fact, the relationships are based on the join paths of the rules as they present the possibilities of join operations. Given a rule $r_{t}$ with join path $J_{t}$, we call a join path as a sub-join path of $J_{t}$ if it is a join path that contains a proper subset of relations of $J R_{t}$. We say a rule defined on a sub-join path of $J_{t}$ is a relevant rule to $r_{t}$. A rule $r_{t}$ can be locally generated only by combining the information from its relevant rules, otherwise, the generated rule contains extra information from relations not in $J_{t}$. Based on the relevance relationship, the rules are organized in a graph structure. Each node in such structure is a rule marked by its join path. Rules in such structure are put into different levels, and the level is determined by the length of its join path. Two nodes are connected if one is the relevant rule of the other. For instance, figure 2 shows a graph structure. $J_{2}$ is a sub-path of $J_{6}$, and $r_{2}$ is a relevant rule to $r_{6}$. They are connected in the graph, and they are on different levels as $J_{2}$ has length 2 and $J_{6}$ has length 3 .

\section{Consistency Checking Algorithm}

Given a set of rules, our goal is to generate the consistent closure of it. Our algorithm uses the join attribute hierarchy property and join groups to efficiently generate the consistent closure. The rules are first divided into different join groups and consistent join groups are generated. Next, based on the join attribute hierarchy, each join attribute is considered for deriving further rules, and any such rules are added to the rule closure. When this procedure terminates, we have the entire consistent closure.

\section{A. Consistent join group generation}

The first step is to generate the consistent join group. With the input as a join group of some given rules, the 


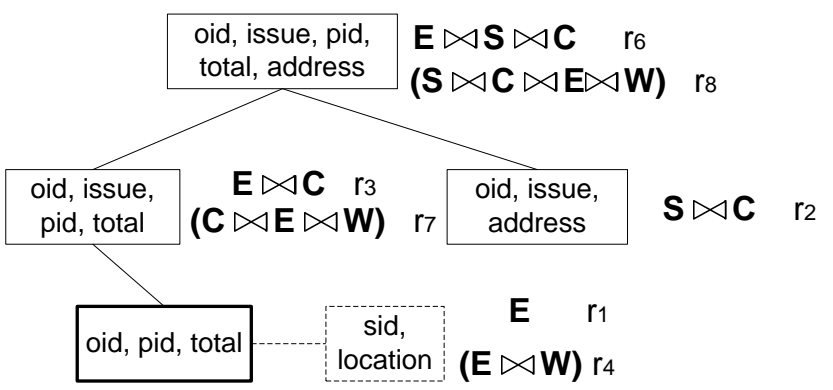

Fig. 2. The consistent join group of oid

algorithm considers each derived rule in the order of join path length. When counting the join path length for a group, we only include the basic relations whose key attributes are the attribute associated with the join group, and we call these relations as dependent relations of the group. A join path that involves only dependent relations is called a dependent join path. Relations whose key attributes are not this attribute are called optional relations. Optional relations or join paths are associated with the dependent join paths. In the graph, we only assign one node for each dependent join path. If the given rule set includes two or more rules that have the same dependent join path, they are assigned to the same node in the graph but identified with their optional relations. When generating the consistent join group on the higher level parent nodes of this node, the algorithm needs to generate corresponding rules using each of the rule associated with this node. We will use our running example to illustrate this.

The join paths discussed below to generate the consistent join group are all dependent join paths. The algorithm looks for each join path length to check if a pair of rules can be joined to form a join path of desired length. Starting from the length of 2, the algorithm takes rules with length less than 2 and generates all the pairs of them. If the resulting rule is not present in the given join group, the algorithm adds it to the group. Otherwise, the resulting rule is merged with the existing rule on their attribute sets. Meanwhile, the graph structure is also built and edges are added between the resulting rule and the rules being examined.

Next, the algorithm checks join path length of 3 to $k$ where $k$ is the number of dependent relations in the join group. When inspecting the length $i$ join-path, the algorithm first takes the rule $r_{m}$ with maximal length $(m<i)$ in the current join group. The algorithm then looks for possible pairs including $r_{m}$, so the other rule $r_{j}$ whose dependent join path should have the property that $\left|J R_{j} \backslash J R_{m}\right|+\left|J R_{m}\right|=i$. The rules are chosen in the reverse order of join path length since the rule with longer join path includes all the attributes from its relevant rules. All the rules with join paths that do not satisfy this property will not be considered in pair with $r_{m}$, and a rule is never paired with its own relevant rules. By iterating over all the join path lengths, the consistent join group can be generated.

To illustrate the process, we use the running example. The first 4 rules have the same key attribute oid, and they are put into the same join group of oid. Within these rules, $r_{4}$ has an optional relation $W$ which does not depend on oid. It is only counted as join path of length 1 and is associated with the node of $r_{1}$ since its dependent join path is the same as $J_{1}$. Then the algorithm begins with join path length of 2 . As the only rule with join path length less than 2 is $r_{1}$, no pair is found. However, the given rules $r_{2}$ and $r_{3}$ are both of length 2 , so they are checked with $r_{1}$ to see the relevance relationship. Thus, $r_{3}$ is connected with $r_{1}$ in the graph. Next, the algorithm checks the length of 3 . Since this join group only includes 3 different relations $\{E, C, S\}$, this is the maximal length to check. The algorithm first takes $r_{2}$ and looks for the rule can pair with it. Among the join path $J_{1}$ and $J_{3}, J_{3}$ is selected since its length is longer, and there is no need to further check with $J_{1}$ as it is relevant to $J_{3}$. Therefore, a rule $r_{6}$ with join path $E \bowtie C \bowtie S$ is added to the join group with the attribute set $A_{2} \bigcup A_{3}$. In the graph structure, this rule is connected with both $r_{2}$ and $r_{3}$.

In addition, rule $r_{4}$ has the optional relation $W$, and it is associated with $r_{1}$ in the group. Therefore, all the rules that $r_{1}$ relevant to also have this optional relation. In such case, based on $r_{6}$ and $r_{3}$, another two rules are added into the join group. This makes join group consistent and is listed in Table II. Here the first 4 rules are given and rule 6 to 8 are added by the algorithm to make the join group consistent. The built graph structure is shown in Figure 2. In the figure, the rule numbers are indicated beside the rule join paths, and the dashed box shows the optional relation of $W$. Since $r_{4}$ has the optional relation $E$ and overlaps with $r_{1}$ on dependent join path, all the parent rules of $r_{1}$ which are $r_{3}, r_{6}$ should also have corresponding rules including the optional relation $W$, which are the rules $r_{7}, r_{8}$.

\begin{tabular}{|c|c|c|c|}
\hline Rule No. & Authorized attribute set & Join Path & Party \\
\hline 1 & $\{$ oid, pid, total $\}$ & $E$ & $P_{E}$ \\
\hline 2 & $\{$ oid, issue, address $\}$ & $S \bowtie_{\text {oid }} C$ & $P_{E}$ \\
\hline 3 & $\{$ oid, pid, total, issue $\}$ & $E \bowtie_{\text {oid }} C$ & $\overline{P_{E}}$ \\
\hline 4 & $\begin{array}{l}\text { \{oid, pid, sid, location, to- } \\
\text { tal }\}\end{array}$ & $E \bowtie_{\text {pid }} W$ & $P_{E}$ \\
\hline 6 & $\begin{array}{l}\text { \{oid, pid, total, issue, ad- } \\
\text { dress }\}\end{array}$ & $\begin{array}{llll}E & \bowtie_{\text {oid }} & S & \bowtie_{\text {oid }} \\
C & & & \\
\end{array}$ & $P_{E}$ \\
\hline 7 & $\begin{array}{l}\text { \{oid, pid, total, issue, loca- } \\
\text { tion, sid }\}\end{array}$ & $\begin{array}{ll}C \bowtie_{\text {oid }} E \bowtie_{\text {pid }} \\
W\end{array}$ & $P_{E}$ \\
\hline 8 & $\begin{array}{l}\text { \{oid, pid, total, issue, loca- } \\
\text { tion, sid, address }\}\end{array}$ & $\begin{array}{l}S \bowtie_{\text {oid }} C \bowtie_{\text {oid }} \\
E \bowtie_{\text {pid }} W\end{array}$ & $P_{E}$ \\
\hline
\end{tabular}

TABLE II

GENERATED CONSISTENT JOIN GROUP OF oid

\section{B. Iteration of key attributes}

We take advantage of the key attributes hierarchy property to develop a mechanism that can achieve the consistent closure. As the key attribute hierarchy can be obtained based on the given join schema, and we assume this information is available when the algorithm is being executed.

At the beginning, the algorithm makes an empty set called target rule set, and the algorithm keeps adding rules into this set. At the end, the target rule set is the rule closure we need. 


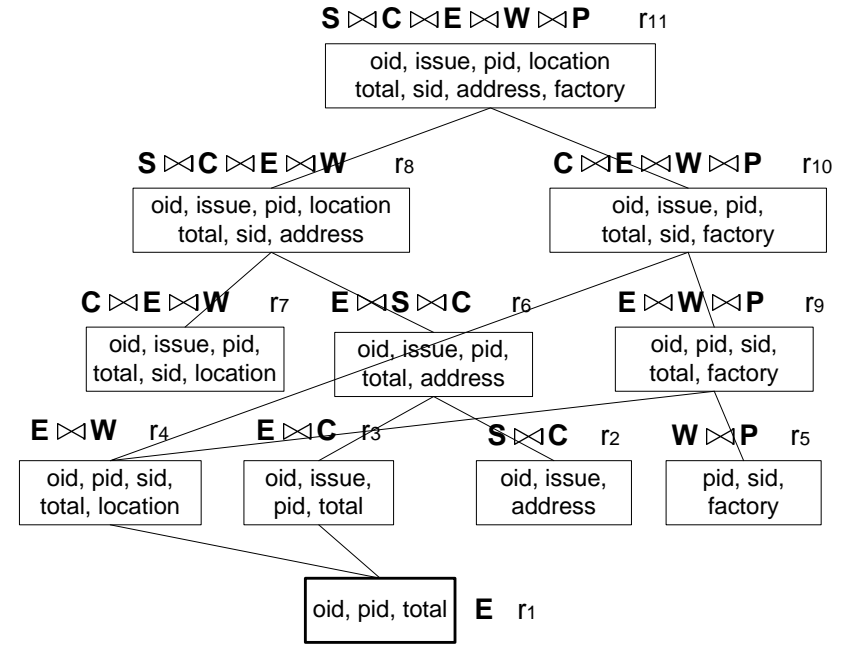

Fig. 3. The graph structure for the consistent closure.

For the given set of rules, the algorithm first puts each rule into different join groups based on its key attribute, and it will only be assigned into one join group. Then, for each join group, the algorithm generates the consistent join group respectively using the mechanism discussed above.

Next, the algorithm iterates each join group according to the level of its associated attribute in the key attribute hierarchy. To begin with, the algorithm inspects the join group of the top level attribute. All the rules in the group being inspected are put into the target rule set first. Then, the algorithm checks the lower level groups one by one. For each join group being checked, all the rules in the current target rule set are iterated. If the rule $r_{t}$ from the current target rule set contains the join attribute that is associated with the join group being checked, then each rule in the join group being checked can join with $r_{t}$. The algorithm generates all these rules by making the union of join paths and the attribute sets, and it adds these generated rules into the target rule set. If there are already a rule in the target rule set with the same join path, the generated rule is merged with the existing rule by making union of the attribute sets from the rules.

As the algorithm iterates all the join groups, the target rule set will keep grow and eventually form the consistent closure. At the time when rules are added to the target rule set, the algorithm also updates the graph structure capturing the rule relevance relationships. If a new rule is generated, it is appended to the graph. Connection edges are added between the rule and the pair of rules that generate it, and the attribute set can be updated. The detail algorithm is described in Alg. 1 .

We can use the running example to illustrate the process of join group iteration. According to the key attribute hierarchy, oid is the top level attribute. Thus, the consistent join group of oid which is listed in Table II is copied to the target rule set. The only remaining join group is the group of pid since there is no given rule takes sid as key attribute. Also, there is only one rule $r_{5}$ in the join group of pid, and this join group is already consistent. As in the key attribute hireachy, pid is on the next level of oid, the algorithm checks each rule in the current target rule set to see if it contains the attribute pid. The set of rules $\left\{r_{1}, r_{3}, r_{4}, r_{6}, r_{7}, r_{8}\right\}$ all have this attribute, so 6 rules joining with $r_{5}$ are generated and added to the target rule set. However, some of these rules have the same join paths and they are merged with existing rules, so only 3 new rules are added to the target rule set. Finally, we generate the consistent closure as listed in Table III. The last three rules are generated in this process. Figure 3 shows the built graph structure, where relevant rules are connected by edges. The attribute sets of the rules are shown in boxes and the join paths together with rule numbers are shown above. The rules are put into 5 levels based on their join path length.

\begin{tabular}{|c|c|c|c|}
\hline Rule No. & Authorized attribute set & Join Path & Party \\
\hline 1 & $\{$ oid, pid, total $\}$ & $E$ & $P_{E}$ \\
\hline 2 & $\{$ oid, issue, address $\}$ & $S \bowtie_{\text {oid }} C$ & $P_{E}$ \\
\hline 3 & $\{$ oid, pid, total, issue $\}$ & $E \bowtie_{\text {oid }} C$ & $P_{E}$ \\
\hline 4 & $\begin{array}{l}\text { \{oid, pid, sid, location, to- } \\
\text { tal\} }\end{array}$ & $E \bowtie_{\text {pid }} W$ & $P_{E}$ \\
\hline 5 & $\{$ pid, sid, factory $\}$ & $W \bowtie_{\text {sid }} P$ & $P_{E}$ \\
\hline 6 & $\begin{array}{l}\text { \{oid, pid, total, issue, ad- } \\
\text { dress }\end{array}$ & $\begin{array}{l}E \bowtie_{\text {oid }} S \bowtie_{\text {oid }} \\
C\end{array}$ & $P_{E}$ \\
\hline 7 & $\begin{array}{l}\text { \{oid, pid, total, issue, loca- } \\
\text { tion, sid }\}\end{array}$ & $\begin{array}{l}E \bowtie_{\text {oid }} C \bowtie_{\text {pid }} \\
W\end{array}$ & $P_{E}$ \\
\hline 8 & $\begin{array}{l}\text { \{oid, pid, total, issue, loca- } \\
\text { tion, sid, address }\}\end{array}$ & $\begin{array}{l}S \bowtie_{\text {oid }} C \bowtie_{\text {oid }} \\
E \bowtie_{\text {pid }} W\end{array}$ & $P_{E}$ \\
\hline 9 & $\{$ oid, pid, sid, factory, total $\}$ & $\begin{array}{l}E \bowtie_{\text {pid }} W \bowtie_{\text {sid }} \\
P\end{array}$ & $P_{E}$ \\
\hline$\overline{10}$ & $\begin{array}{l}\text { \{oid, pid, total, issue, sid, } \\
\text { factory }\}\end{array}$ & $\begin{array}{l}C \bowtie_{\text {oid }} E \bowtie_{\text {pid }} \\
W \bowtie_{\text {sid }} P\end{array}$ & $P_{E}$ \\
\hline 11 & $\begin{array}{l}\{\text { oid, pid, total, issue, loca- } \\
\text { tion, sid, factory, address }\end{array}$ & 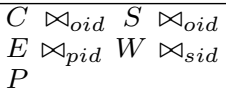 & $P_{E}$ \\
\hline
\end{tabular}

TABLE III

GENERATED CONSISTENT CLOSURE BASED ON GIVEN RULE SET

\section{Average case complexity}

The complexity of the algorithm depends on the given join schema and given rules. In worst case, generating a consistent join group takes exponential time. However, in real cases, usually a join group will not include more than 4 dependent relations. We make the assumption that the maximal number of dependent relations in a join group is 4 . In addition, we assume there are at most $k$ given rules in a join group. Within a join group, there are some given rules overlap on their dependent join paths. Assuming the number of overlapped rules is $p$, then there are $k-p$ nodes for initially given rules. As the most number of relations is 4 , we have $k-p<16$. For the algorithm, at most 22 pairs of nodes will be examined, and there are at most $11+8 p$ rules are added into the consistent join group. As $k$ and $p$ are usually small, the number of rules in a consistent join group is usually less than 20 and the complexity of generating it is also low. we can think the generation of consistent join groups takes constant time and there are at most $C$ rules in a consistent join group.

If there are $m$ join groups in total, it looks like we have the complexity of $C^{m}$ in worst case. However, within a join group, there is only one dependent relation that can join with the rules in the next join group to be inspected. If at most $v$ rules including such dependent relation, then at each step only $v * C$ rules will be added, and the complexity is $O(v * C *(m-1))$. 
In many cases, a join group contains only one or no rule such as the join group of pid and sid in the example, so $C$ is fairly small for many join groups. Also, the length of a valid join path $m$ is usually very small as a join of 5 relations from different enterprises should be a rare case. Therefore, the complexity of the algorithm in real scenario is much lower than the theoretical worst case one.

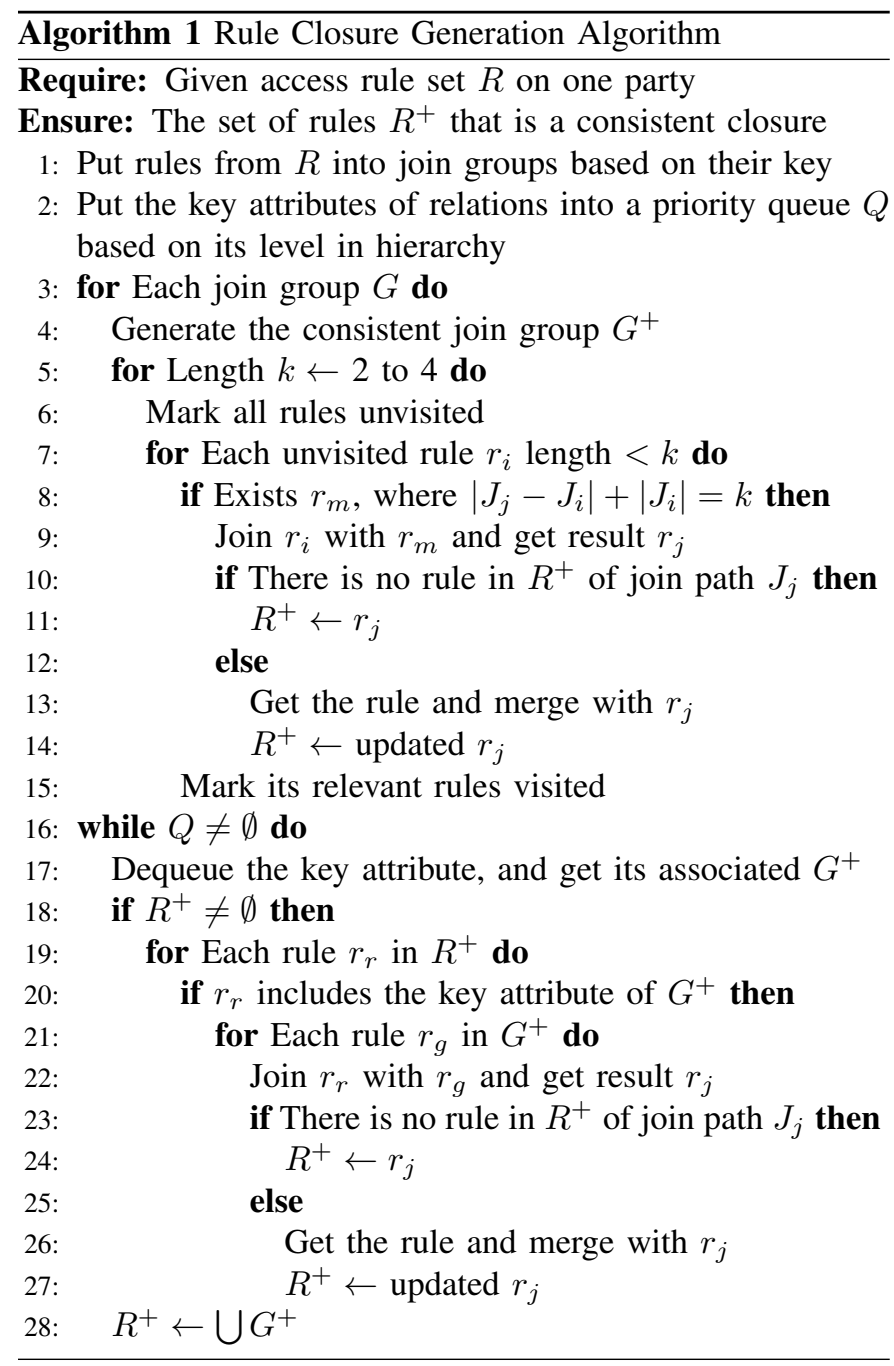

Theorem 1: Given a rule set, the algorithm generates its consistent closure.

Proof: Assuming there are two random rules $r_{i}, r_{j}$, we check whether the consistent closure generated by the algorithm always have $r_{k}$, which is the join result of them. $r_{i}, r_{j}$ can be given rules or the rules generated by the algorithm. Firstly, if $r_{i}, r_{j}$ have the same key attribute, the two rules will be in the same join group. When the algorithm generates the consistent join group, it tries all possible combinations of the dependent relations. In addition, optional relations are considered from bottom up, so there is always a rule in the generated consistent join group that has the same join path as $r_{k}$. When checking the rule relevance in the graph, the attributes from the relevant rules are added to the higher level rules so the rule has the same join path as $r_{k}$ also has all the attributes from $r_{i}$ and $r_{j}$. Since the algorithm examines each join path length in ascending order, it does not matter if $r_{i}, r_{j}$ are given rules or generated rules, and $r_{i}, r_{j}$ are always upwards closed.

If $r_{i}$ and $r_{j}$ are not in the same join group, then we assume the key attribute of $r_{i}$ is on the higher level than the key of $r_{j}$. If both rules are the given rules and $r_{i}$ includes the key attribute of $r_{j}$, when the algorithm iterates the join group of $r_{j}, r_{i}$ is already in the target rule set, and their join result is put into the target rule set. On the other hand, if $r_{i}$ is a generated rule, it is always added into the target rule set by the algorithm. if it can join with $r_{j}$, the result is added to the target rule set also. Thus, after checking the join group of $r_{j}$, all the possible joins over that join attribute are examined. All the rules generated afterwards are joined over the attribute of lower level of $r_{j}$, and rules from these join groups never include the key attribute of $r_{j}$. If $r_{j}$ is a generated rule, it is in its consistent join group, so the algorithm adds the result of $r_{i}$ and $r_{j}$ into the rule set. Therefore, all the rules are upwards closed, and the generated rule set is consistent.

\section{CONSISTENT ACCESS RULE CHANGES}

Cooperative parties may change the access rules over time because of the evolving business needs. The change could either be grant more access privileges to a party or revoke some existing privileges. In addition, the change may cause new conflicts among the rules. Thus, a mechanism is needed to maintain the rule consistency while access rules are changed.

In general, a change of access rule that meet the new business requirement and also has minimal impact on the remaining access rules is the optimal solution. There are different factors can be take into consideration to best recover the rule consistency in the case of change. For instance, according to the business relationships, some access rules maybe more important than the others, so they may have different priorities. In such case, we always prefer to make changes on the less important rules first. Also, in a cooperative environment, some parties collaborate more intimately than the others, and there may also have priorities on different parties. Thus, it is preferred to grant privileges to the intimate parties and revoke privileges from the others. To keep the discussion simple, we propose our mechanism to find the solution that takes minimal changes to the existing access rules in terms of the number rules being modified. The priorities in access rules and parties can be considered by extending such a mechanism, and we leave them for future works.

\section{A. Rule changes}

A possible architecture for the authorization is that the access rules are stored at a central place different from any cooperative parties. An independent query optimizer then read the access rules and generate the query plans. However, cooperative enterprises do not typically share a single independent query optimizer. Instead, each party that answers the queries usually generates the query plan locally. Therefore, without a centralized party, each cooperative party should keep a copy of all access rules locally. We discuss two types of rule changes below. 
1) Independent change: This type of rule change only applies to a single party. Even though a join path involves authorizations from several parties, the change may occur because of a party no longer trusts some other party or their business relationships changed. Such changes usually affects only a small set of rules. Even if the change only takes on a single rule, to maintain the consistency of the rule set, a set of rules may need to be changed accordingly. The discussions below about the granting and revoking of access rules can be directly applied to this type of change. After the party changes its access rules, it broadcasts the change to other cooperative parties.

2) Cooperative change: Sometimes a group of parties may want to update the access rules among them at the same time. These parties may negotiate the rules together and apply the changes on multiple parties at the same time. The group of rules need to be updated as a whole, and we call this type of change as cooperative change. In such case, the updates on several parties need to be synchronized. We call the parties involved in a cooperative rule change as change cooperative parties. A cooperative change need to be performed among these parties atomically from a temporal perspective.

To achieve that, we use $2 \mathrm{PC}$ protocol for the rule update. Among the change cooperative parties, one party is selected as the master party, which we call as coordinator, and all the other change cooperative parties are called slave parties.

Since we assume that the rule changes do not happen frequently, each party can only be involved in one cooperative change process at a time. Therefore, if a slave party is updating its rules, it will have lock them and other rule update requests received are rejected.

Overall, the mechanism works as follows. According to the $2 \mathrm{PC}$ protocol, the update process is divided into a voting phase and a commit phase. In the voting phase, the master party (coordinator) sends messages to all slave parties indicating the set of rules being changed, and each slave party is required to update the rules related to itself. If the slave party can update its rules, which means there is no ongoing rule update at this party, an agreement is sent back to the coordinator, else the request is rejected. Only if the agreements from all slave parties are received, the coordinator will go into the commit phase. In the commit phase, the coordinator sends a commit message to slave parties to finish the rule update and locks are released. Otherwise, the updating transaction is aborted, and the coordinator will try it later.

\section{B. Consistently grant more information}

In the case of rule change, when more access privileges are granted to a party, we need a mechanism to maintain the rule consistency. There are also two types of grants. The first is adding non-key attributes (non-join attributes) to a rule. If a rule is granted with more attributes, then the algorithm first selects the higher level parent rules of this rule in the graph. We search upwards in the graph, and this can be done with a depth first search. If the rule being inspected does not have these expanded attributes, then the algorithm adds these attributes to the rule. If the rule being inspected already has these attributes, the search along this path will stop and another path will be picked. Consequently, the added attributes will be propagated to all the related rules that are at a higher level from the rule being changed. For instance, in our running example, if the attribute delivery is added to $r_{2}$, then the rules $r_{6}, r_{8}, r_{11}$ on the same path need to add this attribute.

In some cases, the attribute added is not the key attribute of the rule being modified, but the attribute is the key attribute for other rules. Therefore, by adding this attribute, the modified rule can possibly further join with other rules. To deal with this situation, once a join attribute is added to a rule (non-key attribute for the rule being modified), the algorithm checks if there exists a join group associated with this attribute. If that is the case, rules which use this attribute as the key attribute are selected from the generated consistent closure. Each rule selected is then joined with the rule being modified, and the resulting rule is added to the rule set or merged with existing rule. Only these rules need to be added to the rule set.

On the other hand, there is another type of change of rules, where a rule on a new join path is granted to a party. In such case, we need to check if this rule can join with existing rules to generate legitimate new rules. The mechanism is similar to the previous approach for generating the consistent closure. As the newly added rule $r_{n}$ has a new join path, we first obtain the key attribute of $r_{n}$, and then $r_{n}$ is put into the join group whose associated attribute is the key attribute of $r_{n}$. Within this group, as a new rule is added, the algorithm recomputes the consistent join group. This can be done efficiently since these rule all can join over their key attributes. In fact, the rule $r_{n}$ is checked with existing rules in the consistent join group. $r_{n}$ is inserted into the graph of the join group, and its relevant rules and the rules it relevant to are not checked with it. All the other rules are checked and $r_{n}$ can join with each of them to form a new rule and put into the consistent join group. The algorithm then keeps the set of newly added rules for the following rule generation.

In the next step, each of the newly added rules is iterated to see what are the other rules that can be generated based on it. For each newly added rule $r_{n}$, the algorithm checks the join attributes in its join path (excluding its key attribute), and for each join attribute the algorithm combines $r_{n}$ with the rules in the join group and add them into the newly added rule set. This process actually finds all needed rules which has the same key attribute as the key of $r_{n}$. After that, the algorithm looks for existing rules that include the key attribute of $r_{n}$ but not using it as their key attributes. Each such rule can join with the newly added rules in the group of $r_{n}$ over the key attribute of $r_{n}$. The algorithm adds all these generated rules into the rule set so as to complete it as a consistent closure. The attribute set of the rules should also be considered. If there exists a rule on the same join path, the attribute sets of the two rules are merged.

In our running example, we can think a new rule $r_{12}$ with join path $E \bowtie_{\text {oid }} S$ is added whose attribute set is \{oid,pid,total,address\}. In this case, the algorithm will put the rule into the join group of oid. In the graph structure, such a rule has relevant rule $r_{1}$, and it is the relevant rule of $r_{6}, r_{8}$. Therefore, other rules in the join group are paired 
with $r_{12}$. However, most of these generated rules already exist in the current join group, so the only new rule $r_{13}$ need to be added is on the join path of $S \bowtie_{\text {oid }} E \bowtie_{\text {pid }} W$. Next, the algorithm checks the rules $r_{12}, r_{13}$. Since both of them include pid as non-key attribute, and there is no join group of sid, both rules are paired with the join group of pid. This results in only one additional rule $r_{14}$ on the join path of $S \bowtie_{\text {oid }} E \bowtie_{\text {pid }} W \bowtie_{\text {sid }} P$. Since oid is the top level join attribute, by adding this rule to the rule set, the consistent rule closure is achieved. Table IV lists these newly added rules.

\begin{tabular}{|c|l|l|l|}
\hline Rule No. & Authorized attribute set & Join Path & Party \\
\hline 12 & \{oid, pid, total, address & $E \bowtie_{\text {oid }} S$ & $P_{E}$ \\
\hline 13 & $\begin{array}{l}\text { \{oid, pid, total, address, sid, } \\
\text { location\} }\end{array}$ & $\begin{array}{l}S \bowtie_{\text {oid }} E \bowtie_{\text {pid }} \\
W\end{array}$ & $P_{E}$ \\
\hline 14 & $\begin{array}{l}\text { \{oid, pid, total, address, sid, } \\
\text { location, factory\} }\end{array}$ & $\begin{array}{l}S \bowtie_{\text {oid }} E \bowtie_{\text {pid }} \\
W \bowtie_{\text {sid }} P\end{array}$ & $P_{E}$ \\
\hline
\end{tabular}

TABLE IV

ACCESS RULES ADDED TOGETHER WITH A RULE GRANT CHANGE

In worst case, if there are already $n$ rules exist in the closure, and there are $C$ rules in the join group. Adding one more rule will need adding additional $C-1$ rules to maintain the consistency. For the above mechanism, the recompilation of the join group will take $C$ steps since each existing rule need to be checked. The remaining complexity depends on the join groups associated with the added rules. If the total number of levels is $u$, and assuming at most $s$ rules in a join group has the join attribute of the inspected group, then the number of pairs to examine in for one join group is $s * C$. The total complexity can be $O(C * u * s)$.

\section{Revocation of existing access rules}

Besides grant of more access privileges, the changes on the rules can also be the revocation of some existing access rules. Similar to the grant case, the revocation can range from removing some non-key attributes to complete removal of a rule. We first discuss the situation where non-key attributes are revoked. The revocation of attributes usually causes inconsistency. Since its relevant rules may still have the revoked attribute, the party can still access these attributes through local computation. Therefore, we need to also revoke these attributes from all relevant rules. Based on the built graph structure, the algorithm retrieves the relevant rules of the rule being modified, if any relevant rules include such revoked attributes, these attributes are also revoked from these rules.

For instance, we can take the example of Figure 3. Let's assume the modification is made on the rule $r_{10}$, and the attribute factory is revoked. In such case, its relevant rules $r_{9}, r_{5}, r_{4}, r_{1}$ are checked. Attribute factory should also be revoked from these rules. Therefore, $r_{9}, r_{5}$ are modified to keep the rule closure consistent.

On the other hand, if a rule with a join path is completely revoked from the rule set, we need to make sure that such a join path can no longer be generated from the remaining relevant rules. Therefore, each possible ways to enforce the join path need to be obtained and the possible pairs should be taken apart. To achieve that, the algorithm uses the graph structure built before. In the graph, only the direct relevant rules of the revoked rule $r_{v}$ are examined. The direct relevant rules of $r_{v}$ are the relevant ones in the graph that directly connected with $r_{v}$ with one edge. For each of the direct connect rule $r_{d}$, the algorithm computes its matching join path $J_{m}$ for $J_{v}$. The matching join path $J_{m}$ is a join path that $J_{m} \bowtie J_{d}=J_{v}, J_{m} \neq J_{v}$, and $\left|J_{m}\right|$ is the minimal one among such join paths. Given the join schema, $J_{m}$ can be efficiently determined by computing the minimal set of $J R_{m}=J R_{v}-J R_{d}$. If such set does not form a join path that is a sub-path of $J_{m}$, then the matching join path of $r_{d}$ does not exist. Otherwise, the matching join path $J_{m}$ is obtained. In the graph, if a rule containing $J_{m}$ is not found, higher level rules connecting to it are examined, and the one with minimal join path length is selected as $J_{m}$.

As we can check the enforceability of the rules [15], we assume we already know what are the locally enforceable rules. Thus, for each pair of rules selected, the algorithm needs to remove one rule from it so as to make the join path no longer enforceable. If a rule in the pair is not locally enforceable, we prefer to remove it since it does not cause cascade revocations. In contrast, if a rule in the pair is locally enforceable, by removing this rule, we need to make sure all the rules that can compose this one are taken apart. Thus, a cascade of revocation will occur. In addition, when iterating each pair, the algorithm also records the number of appearances of the rules. The rule with most appearances is preferred to be removed since removing one such rule can break several pairs. For the locally enforceable rules that are being removed, the algorithm puts them into a queue so that they are processed in a cascaded manner. In worst case, it checks exponential number of pairs, and half of the existing rules need to be removed from the rule set.

For instance, in figure 3 , the rule $r_{10}$ is completely removed. This rule has three direct relevant rules $\left\{r_{4}, r_{9}, r_{3}\right\} . r_{9}$ is first examined, and its matching join path is $\{C\}$. As $\{C\}$ is not available, $r_{3}$ is paired with $r_{9}$. On the other hand, $r_{3}$ can pair with $r_{5}, r_{9}$, and $r_{4}$ cannot pair with any other rule. Therefore, the algorithm needs to break all the pairs of rules $\left\{\left(r_{3}, r_{5}\right),\left(r_{3}, r_{9}\right)\right\}$. Since $r_{3}$ appears in both pairs, the algorithm will revoke it also, and it is put into the queue. As $r_{3}$ is not locally enforceable, we do not need further revocation. Finally, revoking $r_{10}$ with $r_{3}$ will keep the rule closure consistent.

The above mechanism to remove a rule is complicated and it considers only one next level of rules. Thus, we also consider to remove the rules in another way. A revocation is usually issued by a single party, and this party usually revokes the access rules with its own data. Therefore, when a revocation is issued, it is common for the party to revoke all the rules including its basic relation. If this is the case, the revocation involves a set of rules that all including that basic relation, and the consistent closure is still maintained.

According to this idea, if we want to remove a rule, we can also remove a set of rules containing the same basic relation. Thus, another possible way to consistently revoke a rule can be found. The algorithm can first obtain all the relevant rules of $r_{v}$. For each relevant rule, the algorithm 
records the basic relations appearing in the join path. The basic relation associated with fewest number of rules is then selected, and rules including this basic relation are removed from the set.

Back to our example, suppose that we want to revoke rule $r_{10}$. This mechanism first retrieves its relevant rules which are $\left\{r_{4}, r_{5}, r_{9}, r_{3}, r_{1}\right\}$. These join paths are examined, and the appearances of 4 basic relations are checked and counted. Therefore, relation $C$ appears once, $E$ appears 3 times, $W$ appears 3 times, and $P$ appears twice. Thus, the algorithm tries to remove the rule whose join path has $C$. Consequently, $r_{3}$ is removed, and this result is the same as the previous algorithm for this example. In general, these two mechanisms produce different results.

Here, we argue that the rule closure property is different from the rule enforcement issue. Though removing a set of rules will affect the enforceability of other rules, we only focus on maintaining the rule consistency property here. For the second approach, the complexity is $O(n * t)$, where $n$ is the number of relevant rules, and $t$ is the maximal number of relations in a join path.

\section{RELATED WORK}

The problem of controlled data release among collaborating parties has been studied in [8]. The authorization model in this paper is identical to ours and provides the motivation for our work. Its main contribution is an algorithm to check if a query with a given query plan tree can be safely executed. It assumes all the given rules are already upwards closed. However, this is not the case in reality since access rules are usually formulated without consideration of consistency. Therefore, maintaining the consistency of the set of given rules is a crucial problem, that we address in this work.

In another work [7], the same authors evaluate whether the information release asked by a query is allowed by all the authorization rules given to a particular user. It considers all possible combinations of rules and assumes that the rules are defined in an implicit way. Their solution uses a graph model to find all the possible compositions of the given rules, and checks the query against all the generated authorization rules. In our work, we assume access rules are explicitly given. Data release is prohibited if there is no corresponding rule.

There are some works on the access control in collaborative environments. In [20], the authors examined existing access control models as applied to collaboration, and pointed out the weaknesses of these models. In addition, [11], [18] applied RBAC in the collaborative environments. Reference [6] discussed access control problems in social network, and [13] proposed a web services based mechanism for access control in collaboration. All these access control models are different from the one we are using. In [17], collaboration among enterprises was also studied, but that work focused on different application data and multilevel policies.

Processing distributed queries under protection requirements has been studied in [5], [9], [16]. In these works, data access is constrained by a limited access pattern called binding pattern, and the goal is to identify the classes of queries that a given set of access patterns can support. We have a very different authorization model that involves independent parties who cooperate in the execution of a query. There are also classical works on distributed query processing [3], [14], but they do not deal with constraints made by the data owners.

Our authorization model is similar to the view based authorization, and it is related to the area of answering queries using views [12], [10], [19]. These techniques are useful for query optimization, data integration and so on. Although the given view definitions in these works is similar constraints to our access rules, they consider the queries and views in the form of conjunctive queries and they do not consider the collaboration relationships among different parties. These make our problem different from these works, and we may investigate our problem with conjunctive authorization model in the future. To perform the required join operations, there are services like Sovereign joins [1]. It gets encrypted relations from the participating data providers, and sends the encrypted results to the recipients. Such method is useful to enforce our access rules.

\section{CONCLUSIONS AND FUTURE WORKS}

As more and more enterprises work cooperatively to perform computations, securely providing access to cooperative data is important. We use an authorization model for cooperative data access based on the join results of the relational data. However, in the cooperative environment, access conflicts may arise among the rules made according to business requirements. In this paper, we proposed a mechanism to make the set of cooperative access rules consistent. In addition, we also presented algorithms to maintain the rule consistency in the case of granting and revocation of access privileges.

In the future, we plan to perform experiments with real world cases to extensively evaluate the complexity of the algorithms. We will also look for mechanisms that maintain the rule consistency by removing some of the rules instead of simply adding rules. In addition, we will consider Chinese wall policies [4] to remove the inconsistency by preventing local computations, and devise efficient implementation for it. Moreover, we will further look into the more dynamic situation where not only the rules are changed from time to time, but also parties can join and leave the cooperative environment at different times.

\section{REFERENCES}

[1] R. Agrawal, D. Asonov, M. Kantarcioglu, and Y. Li. Sovereign joins. In Proceedings of the 22nd International Conference on Data Engineering, ICDE 2006, 3-8 April 2006, Atlanta, GA, USA, page 26. IEEE Computer Society, 2006.

[2] A. V. Aho, C. Beeri, and J. D. Ullman. The theory of joins in relational databases. ACM Transactions on Database Systems, 4(3):297-314, 1979.

[3] P. A. Bernstein, N. Goodman, E. Wong, C. L. Reeve, and J. B. Rothnie, Jr. Query processing in a system for distributed databases (SDD-1). ACM Transactions on Database Systems, 6(4):602-625, Dec. 1981.

[4] D. F. C. Brewer and M. J. Nash. The chinese wall security policy. In IEEE Symposium on Security and Privacy, pages 206-214, 1989.

[5] A. Calì and D. Martinenghi. Querying data under access limitations. In ICDE, pages 50-59. IEEE, 2008.

[6] B. Carminati and E. Ferrari. Collaborative access control in on-line social networks. pages 231-240. IEEE, 2011. 
[7] S. De Capitani di Vimercati, S. Foresti, S. Jajodia, S. Paraboschi, and P. Samarati. Assessing query privileges via safe and efficient permission composition. In Proceedings of the 2008 ACM Conference on Computer and Communications Security, CCS 2008, Alexandria, Virginia, USA, October 27-31, 2008, pages 311-322. ACM.

[8] S. De Capitani di Vimercati, S. Foresti, S. Jajodia, S. Paraboschi, and P. Samarati. Controlled information sharing in collaborative distributed query processing. In 28th IEEE International Conference on Distributed Computing Systems, Beijing, China, June 2008. IEEE Computer Society.

[9] D. Florescu, A. Y. Levy, I. Manolescu, and D. Suciu. Query optimization in the presence of limited access patterns. In Proc. 15èmes Journées Bases de Données Avancées, BDA, pages 41-60, 1999.

[10] J. Goldstein and P. Larson. Optimizing queries using materialized views: A practical, scalable solution. pages 331-342.

[11] A. Gouglidis and I. Mavridis. domRBAC: An access control model for modern collaborative systems. Computers \& Security, 31(4):540-556, 2012.

[12] A. Y. Halevy. Answering queries using views: A survey. VLDB Journal, 10(4):270-294, 2001.

[13] A. A. E. Kalam, Y. Deswarte, A. Baïna, and M. Kaâniche. Access control for collaborative systems: A web services based approach. pages 1064-1071. IEEE Computer Society, 2007.

[14] D. Kossmann. The state of the art in distributed query processing. $A C M$ Comput. Surv, 32(4):422-469, 2000.

[15] M. Le, K. Kant, and S. Jajodia. Rule configuration checking in secure cooperative data access. In SafeConfig 2012, Baltimore, USA, 2012.

[16] C. Li. Computing complete answers to queries in the presence of limited access patterns. VLDB Journal, 12(3):211-227, 2003.

[17] E. Y. Li, T. C. Du, and J. W. Wong. Access control in collaborative commerce. Decision Support Systems, 43(2):675-685, 2007.

[18] J. S. Park and J. Hwang. Role-based access control for collaborative enterprise in peer-to-peer computing environments. pages 93-99. ACM, 2003.

[19] R. Pottinger and A. Y. Halevy. Minicon: A scalable algorithm for answering queries using views. VLDB J, 10(2-3):182-198, 2001.

[20] Tolone, Ahn, Pai, and Hong. Access control in collaborative systems. CSURV: Computing Surveys, 37, 2005. 